\title{
Chaos Control and Synchronization of Chaotic System Based upon Adaptive Control Algorithm
}

\author{
Israr Ahmad*, Azizan Bin Saaban, Adyda Binti Ibrahim and Mohammad Shahzad
}

\begin{abstract}
Controlling chaos is stabilizing one of the unstable periodic orbits either to its equilibrium point or to a stable periodic orbit by means of an appropriate continuous signal injected to the system. On the other hand, chaos synchronization refers to a procedure where two chaotic oscillators (either identical or nonidentical) adjust a given property of their motion to a common behavior. This research paper concerns itself with the Adaptive control and synchronization of a new chaotic system with unknown parameters. Based on the Lyapunov direct method (LDM), the Adaptive control techniques (ACTs) are designed in such a way that the trajectory of the new chaotic system is globally stabilized to one of its equilibrium points of the uncontrolled system. Moreover, the Adaptive control law is also applied to achieve the synchronization state of two identical systems and two different chaotic systems with fully unknown parameters. The parameters identification, chaos control and synchronization of the chaotic system have been carried out simultaneously by the Adaptive controller. All simulation results are carried out to corroborate the effectiveness and the robustness of the proposed methodology and possible feasibility for synchronizing two chaotic systems by using Mathematica 9 .
\end{abstract}

Keywords - Chaos Control, Synchronization, Lyapunov Stability Theory, Adaptive Control, Barbalat's Lemma, Chaotic system.

\section{INTRODUCTION}

Chaotic systems are nonlinear deterministic systems that display a complicated, randomly or disorderly behavior and are very sensitive to its initial condition, i.e. a small perturbation in its initial conditions, the current trajectory of the system may lead to significantly different future behavior [1]. This extreme sensitive effect of the system termed as the "Butterfly effect" $[1,2]$. Due to critical dependence upon the initial conditions, it becomes very difficult to make accurate long- term predictions about the system over a given time interval [3]. Despite the complexities of a chaotic attractor, this sensitive dependence upon initial conditions can be exploited to sustain the system about some desired final state by carefully choosing a sequence of small perturbation to a

Manuscript received June 06, 2014, revised September 22, 2014.

Israr Ahmad is with the School of Quantitative Sciences, College of Arts \& Sciences, UUM, Saintok Malaysia and with the College of Applied Sciences Nizwa, Ministry of Higher Education, Sultanate of Oman, email: iak 2000plus@yahoo.com

Azizan Bin Saaban and Adyda Binti Ibrahim are with the School of Quantitative Sciences, College of Arts \& Sciences, UUM, Saintok Malaysia, emails: azizan.s@uum.edu.my, adyda@uum.edu.my.

Mohammad Shahzad is with the College of Applied Sciences Nizwa, Ministry of Higher Education, Sultanate of Oman, email dmsinfinite@gmail.com

doi: $10.11601 /$ ijates.v3i2.90 control perimeter or to stabilize an unstable periodic orbit by means of an appropriate continuous signal injected to the system $[3,4]$. This is the basic idea behind the so-called the "Control of Chaos"; where one of the infinite number of unstable periodic orbits that naturally embedded in a chaotic attractor is stabilized to either an equilibrium point or to a stable periodic orbit [3].

On the other hand, for the last two decades, another challenging application of chaos research has emerged, which is known as chaos synchronization. Synchronizing chaotic systems is a process where two (or more) chaotic oscillators eventually proceed synonymously for different initial conditions in all future states [5]. This means that the dynamical state of one of the oscillator is completely traced by the dynamical state of the other oscillator. Chaos Synchronization is one of the critical issues and has received a considerable interest among researchers in nonlinear sciences for more than two decades [6, 7] after the exceptional work of Pecorra and Carroll [5]. Until now, certain linear and nonlinear techniques have been proposed and applied successfully to achieve control and synchronization of chaotic systems [8-20]. Notable among those, ACTs is one of the effectual techniques for stabilizing and synchronizing chaotic systems [11-14]. No Lyapunov exponents or gain matrix is required for its execution. These characteristics free the designer to focus on the control and synchronization problems, leaving irksome model manipulations [14].

Most of the traditional control and synchronization techniques [15-17] are utilized when the system parameters are known. However, in practice, the uncertainties, like external disturbances or parameter mismatches, may destroy the chaos control and the synchronization behavior might be disregarded completely. Therefore, it is very important to design a feedback controller and parameter update law for the control and synchronization of chaotic system consisting of unknown parameters. ACT has the advantage of stabilizing and synchronizing chaotic systems with unknown system parameters [11]. ACTs have received considerable attention in the last few years due to its efficiency, robustness and simple in designing [12]. Motivated by the above discussions, the main objectives of this present study can be summarized as follows:

a) Extending the approaches in $[11,12]$, a simple and efficient ACTs will be designed to stabilize the chaotic system [18] to its unstable equilibrium at the origin with unknown parameters.

b) Based on the Lyapunov Stability Theory (LST) [19] 
and using the ACTs, a class of appropriate control functions and parameter updated laws will be designed to achieve parameters identification and global chaos synchronization of chaotic system [18].

c) Numerical simulations and graphs will be furnished to show the performance and effectiveness of the proposed schemes.

The rest of the research paper is organized as follows. The problem statement and design of the ACT are presented in section 2. In section 3, the description of the system is given, the new results for stabilizing the uncertain chaotic system [18] are derived, and the ACT is applied to solve the synchronization problem for new chaotic system [18]. In section 4, numerical simulations are carried out to show the efficiency of the proposed approaches and support the analytical results and finally the concluding remarks are then given in section 5 .

\section{THE PROBLEM STATEMENT AND ADAPTIVE FEEDBACK CONTROLLER DESIGN}

\section{A. Problem Statement}

Let us consider a chaotic system described as:

$\dot{x}=g_{1}(x)+A_{1} f_{1}(x)$,

where $x=\left[x_{1}, x_{1}, \ldots ., x_{n}\right]^{T}$ is the corresponding state vector, $g_{1}: R^{n} \rightarrow R^{n}$ as the nonlinear continuous vector function, $A_{1} \in R^{m}$ is the system parameters vector and $f_{1}: R^{n} \rightarrow R^{n \times m}$ is the nonlinear matrix function of system (1).

Let us consider the state function of controlled system (1) to be coupled is given as:

$\dot{y}=g_{2}(y)+A_{2} f_{2}(y)+\mu(t)$,

where $y=\left[y_{1}, y_{1}, \ldots, y_{n}\right]^{T} \in R^{n \times 1}$ is the corresponding state vector, $g_{2} \in C^{1}\left(R^{n}, R^{n}\right)$ is the nonlinear continuous functions, $A_{2} \in R^{n}$ as the n-dimension parameters vector and $f_{2} \in C^{1}\left(R^{n}, R^{n \times k}\right)$ is the nonlinear matrix function of the controlled chaotic system (2) and $\mu(t) \in R^{n \times 1}$ as the feedback controller to the controlled system (2).

As certain stabilization/synchronization algorithms belong to the master-slave systems arrangement. Let us consider the master-slave systems configuration for chaotic system (1) and (2) is described as:

$\dot{x}=g_{1}(x)+A_{1} f_{1}(x)$, (Master system)

$\dot{y}=g_{2}(y)+A_{2} f_{2}(y)+\mu(t)$.

$$
\text { (Slave system) }
$$

By defining the error dynamics as the difference between the master and slave systems (3) given by:

$$
\begin{aligned}
& e_{i}(t)=y_{i}(t)-x_{i}(t), \quad e_{i} \in R^{n}, \\
& \dot{e}(t)=g_{2}(y)+A_{2} f(y)-g_{1}(x)-A_{1} f(x)+\mu(t) \Rightarrow \\
& \dot{e}(t)=g_{2}(y)-g_{1}(x)+A_{2} f(y)-A_{1} f(x)+\mu(t) \Rightarrow \\
& \dot{e}(t)=g_{2}(y)-g_{1}(x)+A_{2} f(y)-A_{1} f(x)+\mu(t) .
\end{aligned}
$$

The $F(x, y, e(t))=g_{2}(y)-g_{1}(x)$ is a continuous vector function that contains linear terms and common parts of the master and slave systems.

\section{B. Design of the Adaptive Feedback Controller (ACT)}

If $g_{1}(x)=g_{2}(y), m=k$ and/or $A_{1}=A_{2}$ then $\boldsymbol{X}$ and $\boldsymbol{Y}$ are the states of two identical (nearly identical) chaotic systems and if, $g_{1}(x) \neq g_{2}(y), m \neq k$ and/or $A_{1} \neq A_{2}$, then $\boldsymbol{X}$ and $\boldsymbol{Y}$ are the states of two nonidentical chaotic systems. For the two chaotic systems in the absence of a proper feedback controller, $\left(\mu_{i}=0\right)$, and for the initial conditions $x_{1 m}(0), x_{2 m}(0), \ldots ., x_{n m}(0) \neq y_{1 s}(0), y_{2 s}(0), \ldots ., y_{n s}(0)$, then the trajectories of the two the chaotic systems will quickly diverge from each other in all future states and will become unsynchronized. Hence the basic role of a proper feedback controller ' $\mu(t)$ ' for the chaos control and synchronization problem is such that the error dynamics of (3) converges to zero for all initial conditions, i.e. $\lim _{t \rightarrow \infty}\left\|e_{i}(t)\right\|=\lim _{t \rightarrow \infty}\left\|y_{i}(t)-x_{i}(t)\right\|=0$ for all $e_{i}(0) \in R^{n}$, then the two systems (3) are said to be stabilized/ synchronized. To achieve stabilization/synchronization asymptotically, let us assume the following theorem.

Theorem 1. Trajectories of the two (identical or nonidentical) chaotic oscillators (3) with unknown parameters for all initial conditions $x_{1 d}(0), x_{2 d}(0), \ldots ., x_{n d}(0) \neq y_{1 r}(0), y_{2 r}(0), \ldots ., y_{n r}(0)$ will be stabilized/synchronized asymptotically with the fallowing appropriate regular stabilizing nonlinear feedback controller: $\mu(t)=G(x, y)+E(e), \quad$ where $G(x, y)$ are the control variables of $\boldsymbol{X}$ and $\boldsymbol{Y}$ $E(e)=-k_{i} e_{i}=-k_{i}\left(y_{i}-x_{i}\right), \quad i=1,2, \ldots, n$ is the control function of $e(t)$ and $k_{i}$ is the coupling strength and a same size with $\mu(t)$.

Proof. Let us consider a positive definite quadratic Lyapunov errors function (PDQLEF) $V(e)$ candidate defined by:

$V(e)=\frac{1}{2}\left(e e^{T}+\bar{A}_{1}^{T} \bar{A}_{1}+\bar{A}_{2}^{T} \bar{A}_{2}\right)$,

where $\hat{A}_{1}$ and $\hat{A}_{2}$ are the estimated vectors of the unknown parameters and $\bar{A}_{1}=A_{1}-\widehat{A}_{1}, \bar{A}_{2}=A_{2}-\widehat{A}_{2}$ [10].

It is also assumed that all the variables of the drive and response systems are available and measureable.

It may be noticed that, $V(e): R^{n} \rightarrow R^{n}$ is a positive semidefinite function by construction. Now if the time derivative of $V(e)$ along the trajectories of (4) becomes less than or equal to zero (i.e. $\dot{V}(e) \leq 0$ is also a negative semidefinite function); then the errors signal will approach to zero as time tends to infinity and the states of both projected master system and slave system (3) are stabilized/synchronized.

Since $\dot{V}(e) \leq 0$ is a negative semidefinite function and we cannot immediately conclude that the origin is asymptotically stable. To achieve asymptotic stability of the 
error dynamics (4), let us introduce the following assumptions and a lemma.

Assumption 1 [20]. The vectors $A_{1}, A_{2}$ of the unknown positive parameters are always bounded.

Assumption 2 [21]. If $e_{1}, e_{2}, \ldots, e_{n} \in L^{2} \cap L^{\infty}<\infty$ and $\dot{e}_{1}, \dot{e}_{2}, \ldots, \dot{e}_{n} \in L^{2}<\infty$, then $e(t)=\left[e_{1}(t), e_{2}(t), \ldots, e_{n}(t)\right]$ is also bounded.

Lemma [22]. Let for a non-increasing function $V(t) \in C^{1}$, $\dot{V}(e)$ is uniformly continuous, negative semidefinite and radially unbounded function with: $V(0)=0, V(t)>0$ for all $t \neq 0$ and $\|t\| \rightarrow \infty \Rightarrow V(t) \rightarrow \infty$ then $\lim _{t \rightarrow \infty} \dot{V}(e)=0$.

\section{NEW CHAOTIC SYSTEM DESCRIPTION}

\section{A. Description of New Chaotic System}

Recently, Chunlai Li, et al [18] have proposed and studied a 3-D autonomous chaotic system on the same construction patterns of Chen, Liu and Qi chaotic systems. Chunlai Li, et.al, have added a quadratic nonlinear term to the first equation of Chen system and proposed a new 3-D chaotic system which is topologically different and have more complex dynamic than those of Chen, Liu and Qi Chaotic Systems. The new system can produce a typical double-wing chaotic attractor. The differential equations describing the dynamics of the considered chaotic system are given as:

$\dot{x}=p(y-x)+y z$,

$\dot{y}=(r-p) x+r y-x z$,

$\dot{z}=-q z+s y^{2}$,

where $x, y, z \in R^{n}$ are the state variables and $p, q, r$ and $s$ are the unknown real parameters of the system. By linearizing the system (6) at the equilibrium point $E_{0}=(0,0,0)$ for the parameters values $p, q, r$ and $s$. The Jacobean matrix is given as:

$J_{(0,0,0)}=\left[\begin{array}{ccc}-p & p & 0 \\ (r-p) & r & 0 \\ 0 & 0 & -q\end{array}\right]$.

The characteristic equation of $J_{(0,0,0)}$ is given as:

$\lambda^{3}+(p+q-r) \lambda^{2}+\left(p^{2}+p q-r q-2 p r\right) \lambda+$

$+p^{2} q-2 p q r=0$.

For the parameters values $p=40, q=5, r=30$ and $s \in[0,10]$ the three eigenvalues are $\lambda_{1}=-33.7225$, $\lambda_{2}=-5$ and $\lambda_{3}=23.7228$.

In continuous nonlinear dynamical systems, the condition for stability is that all the eigenvalues and Lyapunov exponents must be negative. We can see that the two eigenvalues are negative and one eigenvalue is positive $\left(\lambda_{1}, \lambda_{2}<0\right.$ and $\left.\lambda_{3}>0\right)$ with the Lyapunov exponents $L_{1}=3.88, \quad L_{2}=0.00, \quad L_{3}=-25.52 \quad$ [18] respectively, which confirm that the system (7) is globally unstable at the equilibrium point $E_{0}=(0,0,0)$. On any initial condition on one of the negative eigenvector, the orbit will converges to the equilibrium point $E_{0}=(0,0,0)$ through the eigen plane of these two negative eigenvectors but any deviation along $\lambda_{3}>0$ will expand and the orbit becomes unstable which shows a saddle point. Hence from the Lyapunov Stability Theory (LST) [19], the equilibrium point $E_{0}=(0,0,0)$ is unstable. Physically, this result bears the fact that the system can oscillate chaotically and prohibits the existence of stable fixed point motion in the system. Thus the new system [18] exhibits a chaotic attractor for parameters values $p=40$, $q=5, \quad r=30$ and $s \in[0,10]$ and hence the control problem takes place.

For the dynamical properties such as the equilibrium points, the maximum Lyapunov exponents' spectrum analysis, the phase portraits and the bifurcation diagram etc for the system (6), one may refer to [18].

\section{B. Tracking Problem for New Chaotic System}

The main objective of this section is to stabilize the new chaotic system to its equilibrium point at the origin by using the ACT when the parameters of the system [18] are fully unknown. Therefore, let us consider the controlled chaotic system (6) described as:

$$
\begin{aligned}
& \dot{x}=p(y-x)+y z+\mu_{1}, \\
& \dot{y}=(r-p) x+r y-x z+\mu_{2}, \\
& \dot{z}=-q z+s y^{2}+\mu_{3},
\end{aligned}
$$

where $x, y, z \in R^{n}$ are the state variables of the system and $\mu(t)=\left[\mu_{1}(t), \mu_{2}(t), \mu_{3}(t)\right]^{T}$ as the stabilizing feedback controller that yet to be designed using the states and estimates of unknown parameters of the uncertain chaotic system.

In the absence of a proper feedback controller, $\left(\mu_{i}(t)=0\right)$,if the initial conditions are $x_{i 1}(0), x_{i 2}(0), \ldots ., x_{i n}(0) \neq y_{j 1}(0), y_{j 2}(0), \ldots ., y_{j n}(0)$ then the trajectories of the chaotic system will quickly bifurcate from each other in all future states and the system will become unstable. Hence, the role of a proper feedback controller for the chaos stabilization problem is to restrict the system converges to $E_{0}=(0,0,0)$ for all initial conditions, then the chaotic system is said to be stabilized at the equilibrium point. Thus the main focus of this part is to stabilize the chaotic system (6) at the origin asymptotically by determining a proper AFC. In order to achieve the global chaos stabilization of system (6), let us assume the following theorem.

Theorem 2. The trajectories of the chaotic oscillator (7) with unknown parameters for all initial conditions $\left(x_{i 1}(0), y_{i 2}(0), z_{i 3}(0) \neq x_{j 1}(0), y_{j 2}(0), z_{j 3}(0)\right)$ will be stabilized asymptotically with the following appropriate regular stabilizing AFC [12] and parameter estimation law given in (15): 
$\mu_{1}(t)=-\hat{p}(y-x)-y z-k_{1} x$

$\mu_{2}(t)=-(\hat{r}-\hat{p}) x-\hat{r} y+x z-k_{2} y$,

$\mu_{3}(t)=-\hat{q} z+\hat{s} y^{2}+k_{3} z$,

where $\hat{p}, \hat{q}, \hat{r}$ and $\hat{s}$ are the estimated value of the unknown parameters $p, q, r$ and $s$ respectively and $k_{i}$ ( $i=1,2,3)$ are the feedback gains.

Proof. Replacing system of equations (8) in (7), we get: $\dot{x}=(p-\hat{p})(y-x)-k_{1} x$,

$\dot{y}=(r-\hat{r}) x-(p-\hat{p}) x+(r-\hat{r}) y-k_{2} y$,

$\dot{z}=-(q-\hat{q}) z+(s-\hat{s}) y^{2}-k_{3} z$.

Let us define the parameter estimation errors as:

$e_{p}=p-\hat{p}, e_{r}=r-\hat{r}, e_{q}=q-\hat{q}, e_{s}=s-\hat{s}$,

$\dot{e}_{p}=-\dot{\hat{p}}, \dot{e}_{r}=-\dot{\hat{r}}, \dot{e}_{q}=-\dot{\hat{q}}, \dot{e}_{s}=-\dot{\hat{s}}$.

Replacing equation (10) in (9), the closed-loop dynamics becomes:

$\dot{x}=e_{p}(y-x)-k_{1} x$,

$\dot{y}=\left(e_{r}-e_{p}\right) x+e_{r} y-k_{2} y$,

$\dot{z}=-e_{q} z+e_{s} y^{2}-k_{3} z$.

For determination of the updated law for adjusting the estimation of the parameters, Let us construct a PDQLEF candidate [12] $V(e)$ defined by:

$V(e)=\frac{1}{2}\left(x^{2}+y^{2}+z^{2}+e_{p}^{2}+e_{r}^{2}+e_{q}^{2}+e_{s}^{2}\right) \geq 0$,

Now the time derivative of the Lyapunov error function (LEF) along the trajectory of the closed loop system (12) is given as:

$\dot{V}(e)=\left(x \dot{x}+y \dot{y}+z \dot{z}+e_{p} \dot{e}_{p}+e_{r} \dot{e}_{r}+e_{q} \dot{e}_{q}+e_{s} \dot{e}_{s}\right)$,

$\dot{V}(t)=-k_{1} x^{2}-k_{2} y^{2}-k_{3} z^{2}+e_{p}((y-x) x-x y-\dot{\hat{p}})+$

$+e_{r}((x+y) y-\dot{\hat{r}})+e_{q}\left(-z^{2}-\dot{\hat{q}}\right)+e_{s}\left(y^{2} z-\dot{\hat{s}}\right)$,

Let us estimate the parameters $\hat{p}, \hat{q}, \hat{r}$ and $\hat{s}$ by the following update law:

$\dot{\hat{p}}=(y-x) x-x y+k_{4} e_{p}, \dot{\hat{r}}=(x+y) y+k_{5} e_{r}$,

$\dot{\hat{q}}=-z^{2}+k_{6} e_{q}, \dot{\hat{s}}=y^{2} z+k_{7} e_{s}$.

Therefore using (15) $\dot{V}(e)=-k_{1} x^{2}-k_{2} y^{2}-k_{3} z^{2}-k_{4} e_{p}{ }^{2}-$ $-k_{5} e_{r}^{2}-k_{6} e_{q}^{2}-k_{7} e_{s}^{2} \leq 0$, which is a negative semidefinite function on $R^{7}$ if $k_{i} \leq 0$ for $i=1,2 ., . ., 7$. Hence the error dynamics is asymptotically stable at the origin.

\section{Identical Synchronization of new Chaotic System with Parameters Estimation}

Most of the synchronization algorithms belong to the masterslave system arrangement. By master-slave system arrangement means that the two chaotic oscillators are coupled in such a way in which one chaotic oscillator is forced to track the other oscillator and the states of two chaotic oscillators show uniform behavior. In order to observe the identical synchronization for chaotic system (6), let us consider the master-slave systems arrangement is described as:

$\dot{x}_{1}=p\left(y_{1}-x_{1}\right)+y_{1} z_{1}$,

$\dot{y}_{1}=(r-p) x_{1}-x_{1} z_{1}+r y_{1}$, (Master System)

$\dot{z}_{1}=-q z_{1}+s y_{1}^{2}$,

and

$\dot{x}_{2}=p\left(y_{2}-x_{2}\right)+\eta_{1}$,

$\dot{y}_{2}=(r-p) x_{2}-x_{2} z_{2}+r y_{2}+\eta_{2}$, (Slave System)

$\dot{z}_{2}=-q z_{2}+s y_{2}{ }^{2}+\eta_{3}$,

where $x_{1}, y_{1}, z_{1} \in R^{n}$ and $x_{2}, y_{2}, z_{2} \in R^{n}$ are the state variables and $p, q, r$ and $s$ of the master and slave systems respectively and $\eta(t)=\left[\eta_{1}(t), \eta_{2}(t), \eta_{3}(t)\right]^{T} \in R^{3 \times 1}$ as the stabilizing nonlinear feedback control function introduced to the slave system that is yet to be designed. By defining the synchronization error dynamics as the difference between the master and slave systems given by $e_{i}(t)=y_{i}(t)-x_{i}(t), e_{i}(t) \in R^{n}$ for $i=1,2,3$.

Thus the time varying error dynamics for the master (15) and slave (16) systems yields as:

$\dot{e}_{1}=p\left(e_{2}-e_{1}\right)+y_{2} z_{2}-y_{1} z_{1}+\eta_{1}$,

$\dot{e}_{2}=(r-p) e_{1}+r e_{2}+\left(x_{1} z_{1}-x_{2} z_{2}\right)+\eta_{2}$,

$\dot{e}_{3}=-q e_{3}+s\left(y_{2}^{2}-y_{1}^{2}\right)+\eta_{3}$.

The aim of the synchronization problem is to design a feedback controller ' $\eta(t)$ ' so that the difference between the trajectories of the two coupled chaotic systems (16) and (17) becomes zero for all future states in the course of time, i.e. $\lim _{t \rightarrow \infty}\|e(t)\|=0$, where $e(t)=\left[e_{1}(t), e_{2}(t), e_{3}(t)\right]^{T}$.

The main focus of this section is to investigate and study the synchronization problem of two identical chaotic systems (16) and (17) with fully unknown parameters. It is clear that the synchronization of two chaotic systems is equivalent to the problem of stabilizing the system (14) at the equilibrium point $(0,0)$ by determining a suitable feedback controller ' $\eta(t)$ ' and parameter estimation and proper updated laws for system of equations (18) so that when synchronizing the two chaotic systems (16) and (17), the effect of nonlinearity of chaotic systems does not neglect and the error dynamics (18) of the two identical chaotic systems (16) and (17) converges to the equilibrium point asymptotically with less control effort and sufficient transient speed. For these motivations, we assume the following theorem.

Theorem 3. The trajectories of the two identical chaotic systems (16) and (17) for all initial conditions $\left(x_{1 m}(0), x_{2 m}(0), x_{3 d}(0) \neq y_{1 s}(0), y_{2 s}(0), y_{3 s}(0)\right) \quad$ will $\quad$ be synchronized asymptotically with fully unknown parameters by the fallowing appropriate regular stabilizing AFC functions and parameter estimation law given in (26) as: 
$\eta_{1}(t)=-\hat{p}\left(e_{2}-e_{1}\right)+y_{1} z_{1}-y_{2} z_{2}-k_{1} e_{1}$,

$\eta_{2}(t)=-(\hat{r}-\hat{p}) e_{1}+x_{2} z_{2}-x_{1} z_{1}-\hat{r} e_{2}-k_{2} e_{2}$,

$\eta_{3}(t)=\hat{q} e_{3}-\hat{s}\left(y_{2}^{2}-y_{1}^{2}\right)-k_{3} e_{3}$,

where $\hat{p}, \hat{q}, \hat{r}$ and $\hat{s}$ are the estimated value of the unknown parameters $p, q, r$ and $s$ respectively and $k_{i}$ are the control gains.

Proof. Replacing system of equation (19) in (18), we get:

$\dot{e}_{1}=(p-\hat{p})\left(e_{2}-e_{1}\right)-k_{1} e_{1}$,

$\dot{e}_{2}=(r-\hat{r}) e_{1}-(p-\hat{p}) e_{1}+(r-\hat{r}) e_{2}-k_{2} e_{2}$,

$\dot{e}_{3}=-(q-\hat{q}) e_{3}+(s-\hat{s})\left(y_{2}^{2}-y_{1}^{2}\right)-k_{3} e_{3}$.

Let us define the parameter estimation errors as:

$e_{p}=p-\hat{p}, e_{r}=r-\hat{r}, e_{q}=q-\hat{q}, e_{s}=s-\hat{s}$,

$\dot{e}_{p}=-\dot{\hat{p}}, \dot{e}_{r}=-\dot{\hat{r}}, \dot{e}_{q}=-\dot{\hat{q}}, \dot{e}_{s}=-\dot{\hat{s}}$.

Substituting equation (21) in (20), the error dynamics can be described as:

$\dot{e}_{1}=e_{p}\left(e_{2}-e_{1}\right)-k_{1} e_{1}$,

$\dot{e}_{2}=\left(e_{r}-e_{p}\right) e_{1}+e_{r} e_{2}-k_{2} e_{2}$,

$\dot{e}_{3}=-e_{q} e_{3}+e_{s}\left(y_{2}^{2}-y_{1}^{2}\right)-k_{3} e_{3}$,

For determination of the updated law for adjusting the estimation of the parameters, let us construct the same PDQLEF candidate as in (13):

$V(e)=\frac{1}{2} \sum_{i=1}^{3}\left(e_{i}^{T} e_{i}\right)+\frac{1}{2}\left(e_{p}^{2}+e_{r}^{2}+e_{q}^{2}+e_{s}^{2}\right) \geq 0$.

Now the time derivative of the LEF along the direction of the error dynamics (23) as:

$$
\begin{aligned}
& \dot{V}(e)=\left(e_{1} \dot{e}_{1}+e_{2} \dot{e}_{2}+e_{3} \dot{e}_{3}+e_{p} \dot{e}_{p}+e_{r} \dot{e}_{r}+e_{q} \dot{e}_{q}+e_{s} \dot{e}_{s}\right), \\
& \dot{V}(e)=-k_{1} e_{1}^{2}-k_{2} e_{2}^{2}-k_{3} e_{3}^{2}-e_{p}\left(e_{1}^{2}+\dot{\hat{p}}\right)- \\
& -e_{r}\left(-e_{2}\left(e_{1}+e_{2}\right)+\dot{\hat{r}}\right)-e_{q}\left(e_{3}^{2}+\dot{\hat{q}}\right)- \\
& -e_{s}\left(-e_{3}\left(y_{2}^{2}-y_{1}^{2}\right)+\dot{\hat{s}}\right) .
\end{aligned}
$$

When the master system's parameters are not available, then we can achieve parameters identification and synchronization simultaneously with the controlled slave system and the parameters estimation update law. Based on (25), the update laws $\hat{p}, \hat{q}, \hat{r}$ and $\hat{s}$ for the parameters estimates is derived according to the following update law:

$\dot{\hat{p}}=-e_{1}^{2}+k_{4} e_{p}, \dot{\hat{r}}=\left(e_{1}+e_{2}\right) e_{2}+k_{5} e_{r}$,

$\dot{\hat{q}}=-e_{3}^{2}+k_{6} e_{q}, \dot{\hat{s}}=\left(y_{2}^{2}-y_{1}^{2}\right) e_{3}+k_{7} e_{s}$.

With this choice of controller (19) and the parameters estimation update laws (26) yields:

$\dot{V}(e)=-\left[\left(\sum_{i=1}^{3} k_{i} e_{i}^{2}\right)+\left(e_{p}^{2}+e_{q}^{2}+e_{r}^{2}+e_{s}^{2}\right)\right] \leq 0$,

i.e. $\dot{V}(e)=-e^{T} P e \leq 0$, Which is a negative semidefinite function on $R^{7}$ [14]. Hence the error dynamics is stable at the origin.
Since from (24) and (27), $a_{1}, b_{1}, c_{1}, d_{1}, \delta_{1}, f_{1}$ and $g_{1}$ are bounded [20], as $e_{1}, e_{2}, e_{3} \in L^{2} \cap L^{\infty}<\infty \quad$ and $\dot{e}_{1}, \dot{e}_{2}, \dot{e}_{3} \in L^{2}<\infty$. It can be easily check that $\ddot{e}(t)$ is bounded. Thus from (20) $\left[e_{1}(t), e_{2}(t), e_{3}(t)\right]$ is also bounded [21]. As the trajectories of chaotic systems are always bounded [23], so from (19), $\eta(t)$ is also bounded. Therefore, if $\lambda_{\min }(P)$ is the minimum eigenvalue of a positive definite matrix $P$, then by Barbalat's lemma [22]:

$\int_{0}^{t} \lambda_{\min }(P)\|e\|^{2} d t \leq \int_{0}^{t}\left(e^{T} P e\right) d t=\int_{0}^{t}-\dot{V}(t) d t=V(0)-V(t) \leq 0$.

This implies that, $\lim _{t \rightarrow \infty}\left\|e_{i}(t)\right\|=0$, for $i=1,2,3$.

Hence the equilibrium point (origin) is asymptotically stable. Thus by the LST [19], it is evident that the synchronization errors and parameter estimation errors are decay to zero asymptotically with the course of time for all initial conditions. Hence the two coupled chaotic systems (16) and (17) with unknown parameters are asymptotically synchronized.

\section{Chaos Synchronization of Nonidentical Chaotic Systems with Parameters Estimation}

In order to observe the switching synchronization for chaotic system (5), it is assumed that the new chaotic system drives the Liu chaotic system [24]. Therefore the master is described as:

$\dot{x}_{1}=p\left(y_{1}-x_{1}\right)+y_{1} z_{1}$,

$\dot{y}_{1}=(r-p) x_{1}+r y_{1}-x_{1} z_{1}$, (Master system)

$\dot{z}_{1}=-q z_{1}+s y_{1}^{2}$,

and the slave system, we consider the controlled Liu chaotic system as:

$\dot{x}_{2}=\alpha\left(y_{2}-x_{2}\right)+\eta_{1}$,

$\dot{y}_{2}=\beta x_{2}-x_{2} z_{2}+\eta_{2}, \quad$ (Slave system)

$\dot{z}_{2}=-\gamma z_{2}+\delta x_{2}^{2}+\eta_{3}$,

where $x_{1}, y_{1}, z_{1} \in R^{n} \quad$ and $\quad x_{2}, y_{2}, z_{2} \in R^{n} \quad$ are the corresponding state vectors of master and slave systems respectively, $p, q, r$ and $s$ are the system parameters of the master system and $\alpha, \beta, \gamma$ and $\delta$ are the system parameters of the slave system respectively and $\eta(t)=\left[\eta_{1}(t), \eta_{2}(t), \eta_{3}(t)\right]^{T} \in R^{n \times 1}$ as the AFC that yet to be designed. The error dynamics for the master (27) and slave (28) systems can be described as:

$\dot{e}_{1}=\alpha\left(y_{2}-x_{2}\right)-p\left(y_{1}-x_{1}\right)-y_{1} z_{1}+\eta_{1}$,

$\dot{e}_{2}=\beta x_{2}-(r-p) x_{1}-r y_{1}+x_{1} z_{1}-x_{2} z_{2}+\eta_{2}$,

$\dot{e}_{3}=-\gamma z_{2}+q z_{1}+\delta x_{2}^{2}-s y_{1}^{2}+\eta_{3}$.

The main contribution of this section is to achieve asymptotic synchronization between two different chaotic systems (27) and (28) with fully unknown parameters such that the synchronization errors and parameter estimation errors are decay to zero with the course of time for all initial 
conditions: $\left(x_{1 d}(0), x_{2 d}(0), \ldots ., x_{n d}(0) \neq y_{1 r}(0), y_{2 r}(0), \ldots ., y_{n r}(0)\right)$; by designing such a feedback adaptive controller that the Liu system [24] force to track the new system [18] and the two systems show similar behavior for all future states. To achieve these objectives, let us assume the following theorem.

Theorem 4. The trajectories of the two nonidentical chaotic systems (27) and (28) for all initial conditions $\left(x_{1 m}(0), x_{2 m}(0), x_{3 d}(0) \neq y_{1 s}(0), y_{2 s}(0), y_{3 s}(0)\right) \quad$ will $\quad$ be synchronized asymptotically with fully unknown parameters by the fallowing appropriate regular stabilizing AFC functions and parameter estimation law given in (37) as:

$$
\begin{aligned}
& \eta_{1}(t)=-\hat{\alpha}\left(y_{2}-x_{2}\right)+\hat{p}\left(y_{1}-x_{1}\right)+y_{1} z_{1}-k_{1} e_{1}, \\
& \eta_{2}(t)=-\hat{\beta} x_{2}+(\hat{r}-\hat{p}) x_{1}+\hat{r} y_{1}+x_{2} z_{2}-x_{1} z_{1}-k_{2} e_{2}, \\
& \eta_{3}(t)=\hat{\gamma} z_{2}-\hat{q} z_{1}-\hat{\delta} x_{2}{ }^{2}+\hat{s} y_{1}^{2}-k_{3} e_{3},
\end{aligned}
$$

where $\hat{p}, \hat{q}, \hat{r}$ and $\hat{s}$, and $\hat{\alpha}, \hat{\beta}, \hat{\gamma}$ and $\hat{\delta}$ are the estimated value of the unknown parameters $p, q, r$ and $s$, and $\alpha, \beta, \gamma$ and $\delta$ respectively and $k_{i}$ are the feedback gains.

Proof. Replacing system of equations (30) in (29), the error dynamics can be expressed as:

$$
\begin{aligned}
& \dot{e}_{1}=(\alpha-\hat{\alpha})\left(y_{2}-x_{2}\right)-(p-\hat{p})\left(y_{1}-x_{1}\right)-k_{1} e_{1}, \\
& \dot{e}_{2}=(\beta-\hat{\beta}) x_{2}-(r-\hat{r}) x_{1}+(p-\hat{p}) x_{1}-(r-\hat{r}) y_{1}-k_{2} e_{2}, \\
& \dot{e}_{3}=-(\gamma-\hat{\gamma}) z_{2}+(q-\hat{q}) z_{1}+(\delta-\hat{\delta}) x_{2}{ }^{2}-(s-\hat{s}) y_{1}{ }^{2}-k_{3} e_{3} .
\end{aligned}
$$

Let us define the parameter estimation errors as:

$$
\begin{aligned}
& e_{p}=p-\hat{p}, e_{r}=r-\hat{r}, e_{q}=q-\hat{q}, e_{s}=s-\hat{s}, \\
& e_{\alpha}=\alpha-\hat{\alpha}, e_{\beta}=\beta-\hat{\beta}, e_{\gamma}=\gamma-\hat{\gamma}, e_{\delta}=\delta-\hat{\delta}, \\
& \dot{e}_{p}=-\dot{\hat{p}}, \dot{e}_{r}=-\dot{\hat{r}}, \dot{e}_{q}=-\dot{\hat{q}}, \dot{e}_{s}=-\dot{\hat{s}}, \\
& \dot{e}_{\alpha}=-\dot{\hat{\alpha}}, \dot{e}_{\beta}=-\dot{\hat{\beta}}, \dot{e}_{\gamma}=-\dot{\hat{\gamma}}, \dot{e}_{\delta}=-\dot{\hat{\delta}} .
\end{aligned}
$$

Replacing system of equation (31) in (32), the error dynamics can be described as:

$$
\begin{aligned}
& \dot{e}_{1}=e_{\alpha}\left(y_{2}-x_{2}\right)-e_{p}\left(y_{1}-x_{1}\right)-k_{1} e_{1}, \\
& \dot{e}_{2}=e_{\beta} x_{2}-e_{r} x_{1}+e_{p} x_{1}-e_{r} y_{1}-k_{2} e_{2}, \\
& \dot{e}_{3}=-e_{\gamma} z_{2}+e_{q} z_{1}+e_{\delta} x_{2}^{2}-e_{s} y_{1}^{2}-k_{3} e_{3} .
\end{aligned}
$$

For determination of the updated law for adjusting the estimation of the parameters, let us construct PDQLEF candidate as in (13):

$$
\begin{aligned}
V(e)= & \frac{1}{2} \sum_{i=1}^{3}\left(e_{i}^{T} e\right)+\frac{1}{2}\left(e_{p}^{2}+e_{r}^{2}+e_{q}^{2}+e_{s}^{2}+e_{\alpha}^{2}+\right. \\
& \left.+e_{\beta}^{2}+e_{\gamma}^{2}+e_{\delta}^{2}\right) \geq 0
\end{aligned}
$$

which is a positive definite function on $R^{11}$.

Now the time derivative of the LEF along the trajectories of the error dynamics (34) as:

$$
\begin{aligned}
\dot{V}(e)= & -k_{1} e_{1}^{2}-k_{2} e_{2}^{2}-k_{3} e_{3}^{2}-e_{p}\left(\left(y_{1}-x_{1}\right) e_{1}-e_{2} x_{1}+\dot{\hat{p}}\right)+ \\
& +e_{q}\left(e_{3} z_{1}-\dot{\hat{q}}\right)-e_{r}\left(\left(x_{1}+y_{1}\right) e_{2}+\dot{\hat{r}}\right)-e_{s}\left(e_{3} y_{1}^{2}+\dot{\hat{s}}\right)+ \\
& +e_{\alpha}\left(\left(y_{2}-x_{2}\right) e_{1}-\dot{\hat{\alpha}}\right)+e_{\beta}\left(e_{2} x_{2}-\dot{\hat{\beta}}\right) \\
& -e_{\gamma}\left(e_{3} z_{2}+\dot{\hat{\gamma}}\right)+e_{\delta}\left(e_{3} x_{2}{ }^{2}-\dot{\hat{\delta}}\right) .
\end{aligned}
$$

When the master system's parameters are not available, then we can achieve parameters identification and synchronization simultaneously with the controlled slave system and the parameters estimation update law. Based on (36), the update laws $\hat{p}, \hat{q}, \hat{r}, \hat{s}, \hat{\alpha}, \hat{\beta}, \hat{\gamma}$ and $\hat{\delta}$ for the parameters estimates are derived according to the following update law:

$$
\begin{aligned}
& \dot{\hat{p}}=-\left(y_{1}-x_{1}\right) e_{1}+e_{2} x_{1}+k_{4} e_{p}, \\
& \dot{\hat{q}}=e_{3} z_{1}+k_{5} e_{q}, \dot{\hat{r}}=-\left(x_{1}+y_{1}\right) e_{2}+k_{6} e_{r}, \\
& \dot{\hat{s}}=-e_{3} y_{1}^{2}+k_{7} e_{s}, \\
& \dot{\hat{\alpha}}=\left(y_{2}-x_{2}\right) e_{1}+k_{8} e_{\alpha}, \dot{\hat{\beta}}=e_{2} x_{2}+k_{9} e_{\beta}, \\
& \dot{\hat{\gamma}}=-e_{3} z_{2}+k_{10} e_{\gamma}, \dot{\hat{\delta}}=e_{3} x_{2}^{2}+k_{11} e_{\delta} .
\end{aligned}
$$

With this choice of controller (30) and the updated laws (37), the LEF (36) yields:

$$
\begin{aligned}
\dot{V}(e)= & -\sum_{i=1}^{3}\left(k_{i} e_{i}^{2}\right)-\left(k_{4} e_{p}^{2}+k_{5} e_{r}^{2}+k_{6} e_{q}^{2}+\right. \\
& \left.+k_{7} e_{s}^{2}+k_{8} e_{\alpha}^{2}+k_{9} e_{\beta}^{2}+k_{10} e_{\gamma}^{2}+k_{11} e_{\delta}^{2}\right) \leq 0,
\end{aligned}
$$

i.e. $\dot{V}(e)=-e^{T} P e \leq 0$, Which is a negative semidefinite function on $R^{11}$ and $P=\left(\begin{array}{ccccc}k_{1} & 0 & . & . & 0 \\ 0 & k_{2} & 0 & . & 0 \\ . & . & . & . & . \\ . & . & . & . & . \\ 0 & 0 & . & . & k_{11}\end{array}\right)$. Hence the error dynamics (34) is stable. Using assumption $1 \& 2$, $\left[e_{1}(t), e_{2}(t), e_{3}(t)\right]$ and $\eta(t)$ are bounded. Therefore, if $\lambda_{\text {min }}(P)$ is the minimum eigenvalue of a positive definite matrix $P$, then by Barbalat's lemma [21]:

$\int_{0}^{t} \lambda_{\min }(P)\|e\|^{2} d t \leq \int_{0}^{t}\left(e^{T} P e\right) d t=\int_{0}^{t}-\dot{V}(t) d t=V(0)-V(t) \leq 0$. Thus, the error dynamics (34) converges to equilibrium point asymptotically, i.e. $\lim _{t \rightarrow \infty}\left\|e_{i}(t)\right\|=0$ for $i=1,2,3$.

Hence based on the LST [19], it is obvious that the synchronization errors and parameter estimation errors are decay to zero asymptotically with the course of time for all initial conditions: $\left(x_{i 1}(0), y_{i 2}(0), z_{i 3}(0) \neq x_{j 1}(0), y_{j 2}(0), z_{j 3}(0)\right)$. Thus the two nonidentical chaotic systems (27) and (28) with fully unknown parameters are asymptotically synchronized. 


\section{NUMERICAL SIMULATIONS AND DISCUSSION}

The numerical simulations were carried out using the Mathematica 9. For this numerical simulation, the parameters for the new chaotic system [18] are selected as: $p=40, q=5, r=30, s=3$, with initial conditions: (2, $3,2)$ and $(-5,-7,5)$. The four unknown parameters are taken in simulations so that the new system [18] exhibit chaotic attractor.

For the adaptive and update law, the feedback control gains are taken as: $k_{i}=3$ for $i=1,2, \ldots ., 11$. The parameters for the Liu chaotic system [24] are taken as: $\alpha=10$, $\beta=40, \gamma=2.5, \delta=4$, with initial values: $(8,21,17)$ and $(15,7,6)$.
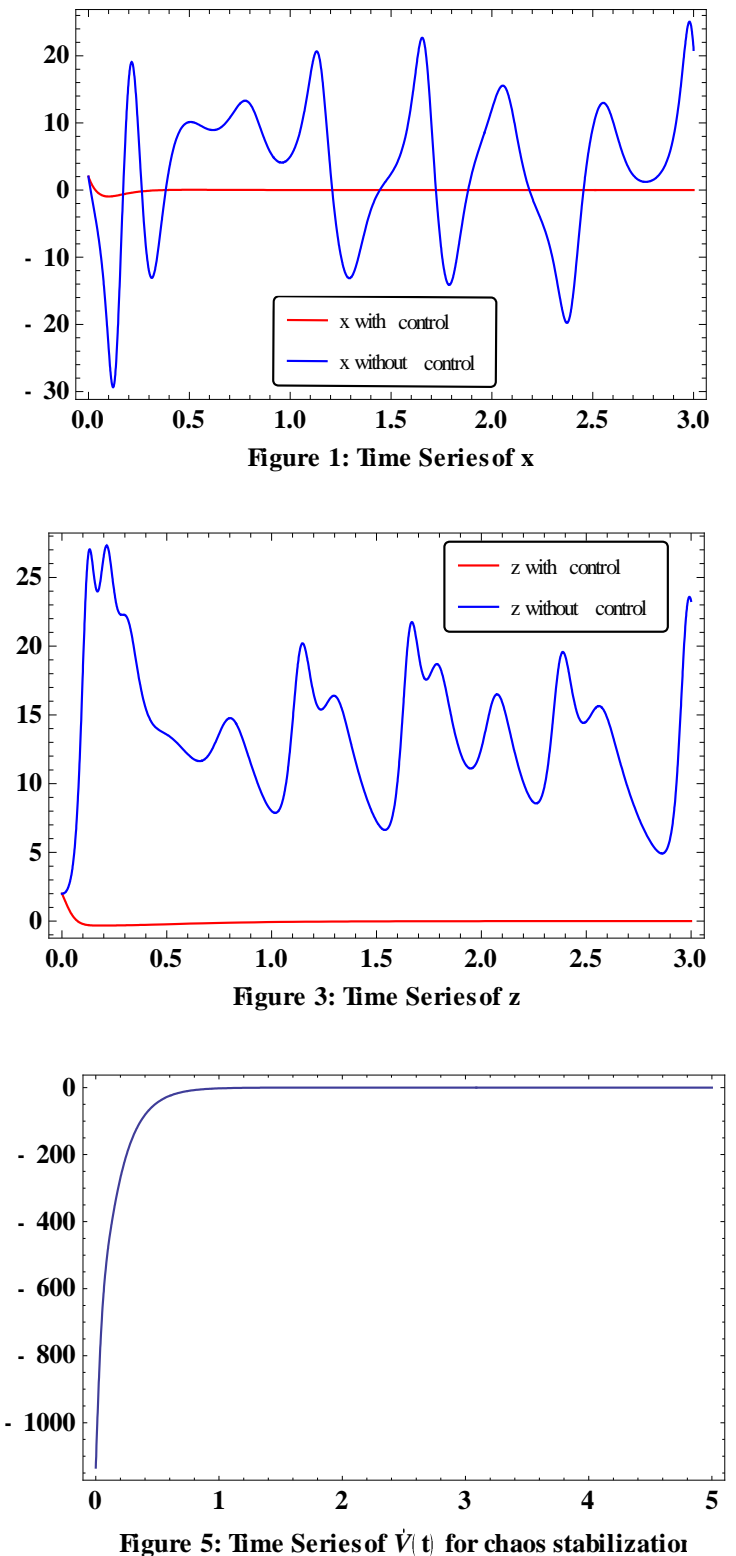

For the chaotic system [18] the time histories of the state vectors of controlled and uncontrolled are provided in Figs. 1 to 3 , which shows the system [18] is well controlled after transient time of $0.2 \mathrm{~s}$; which justified the performance of using the type of control schemes presented in this research work. Figure 4 illustrates the time history of the parameters estimates for suppressing chaos in the new system under the update law (14) and Fig. 9 and 14 shows the time history of the parameters estimates for synchronizing the system [18] with the initial estimated parameters $p_{1}(0)=22$, $q_{1}(0)=10, r_{1}(0)=36$ and $s_{1}(0)=6$. It is clear that the estimations $\hat{p}, \hat{q}, \hat{r}$ and $\hat{s}$ of unknown parameters $p, q, r$, and $s$ converge to $p, q, r$, and $s$ respectively as time goes to infinity under the update laws (26) and (37).
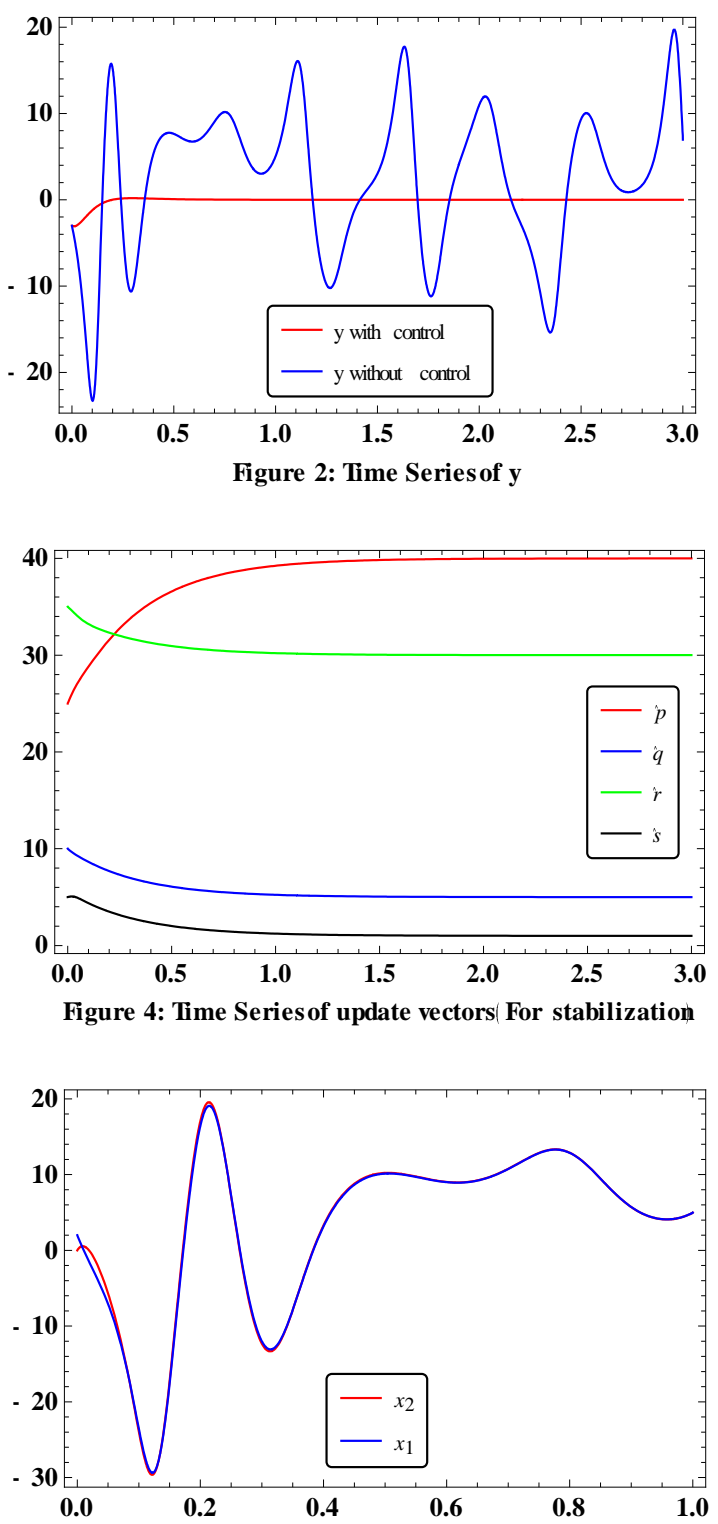

Figure 6: Time Series of $x_{2} \& x_{1}$ for identical synchronization 


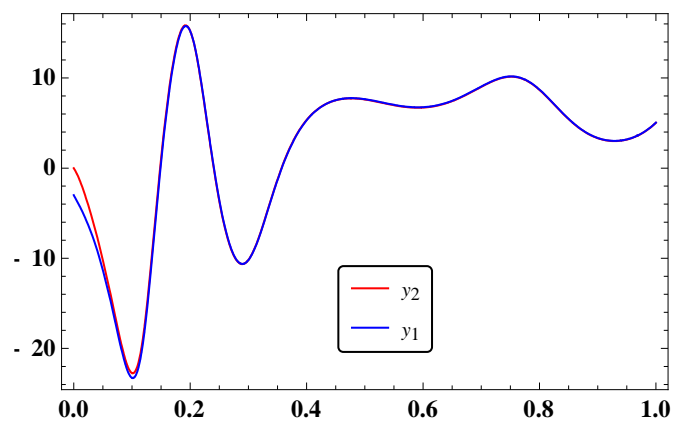

Figure 7: Time Series of $y_{2} \& y_{1}$ for identical synchronizatior
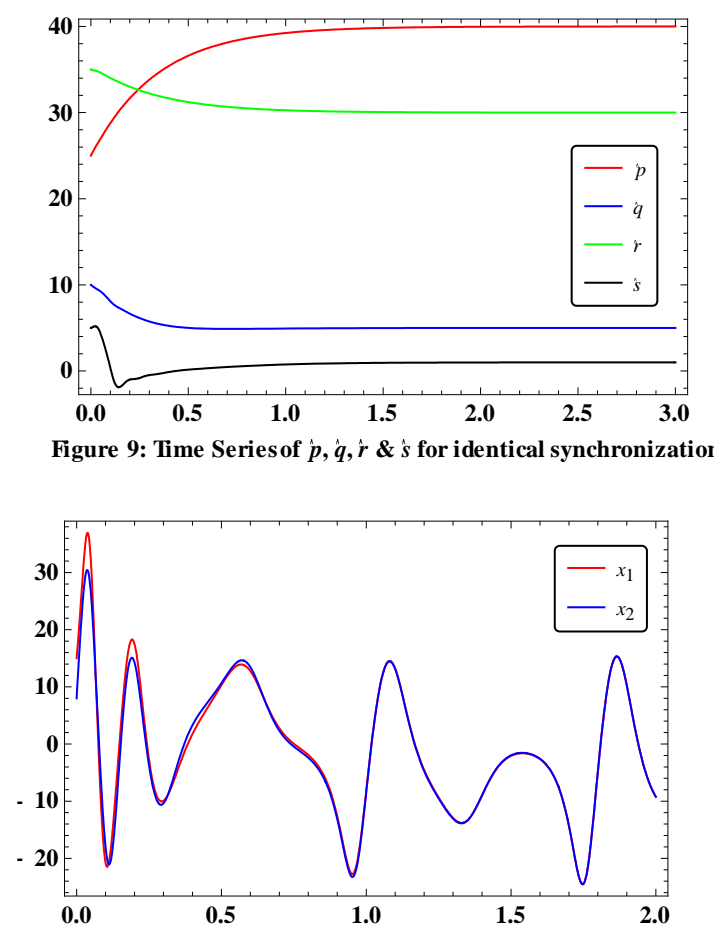

Figure 11: Time Series of $x_{1} \& x_{2}$ for nonidentical synchronizatior

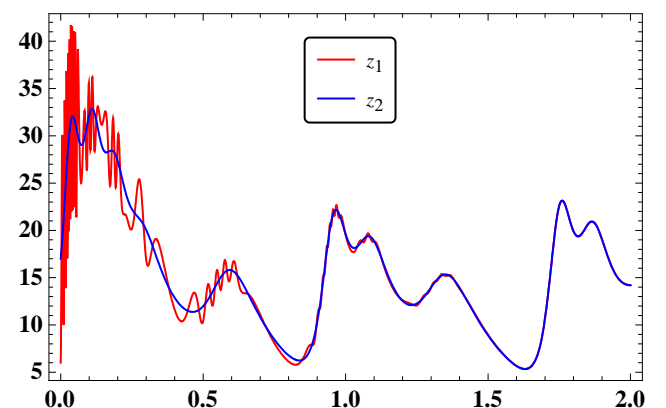

Figure 13: Time Series of $z_{1} \& z_{2}$ for nonidentical synchronizatior

Figure 5 presents the time derivative of the LEF for the controlling of chaos in system [18] that characterizes that the new system is dissipative $(\dot{V}(e)<0)$. The time series of the state vectors of the two identical chaotic systems (16) and (17) to observe the synchronization. Figure 9 depicts the time history of the parameters estimates for synchronization of the two identical chaotic systems [18] under the update law (26). The evolution of the synchronized errors with response system parameters unknown is depicted in Fig. 10. Figures 11-13 elaborate the time history of state vectors of the two nonidentical chaotic systems ([18], [24]).
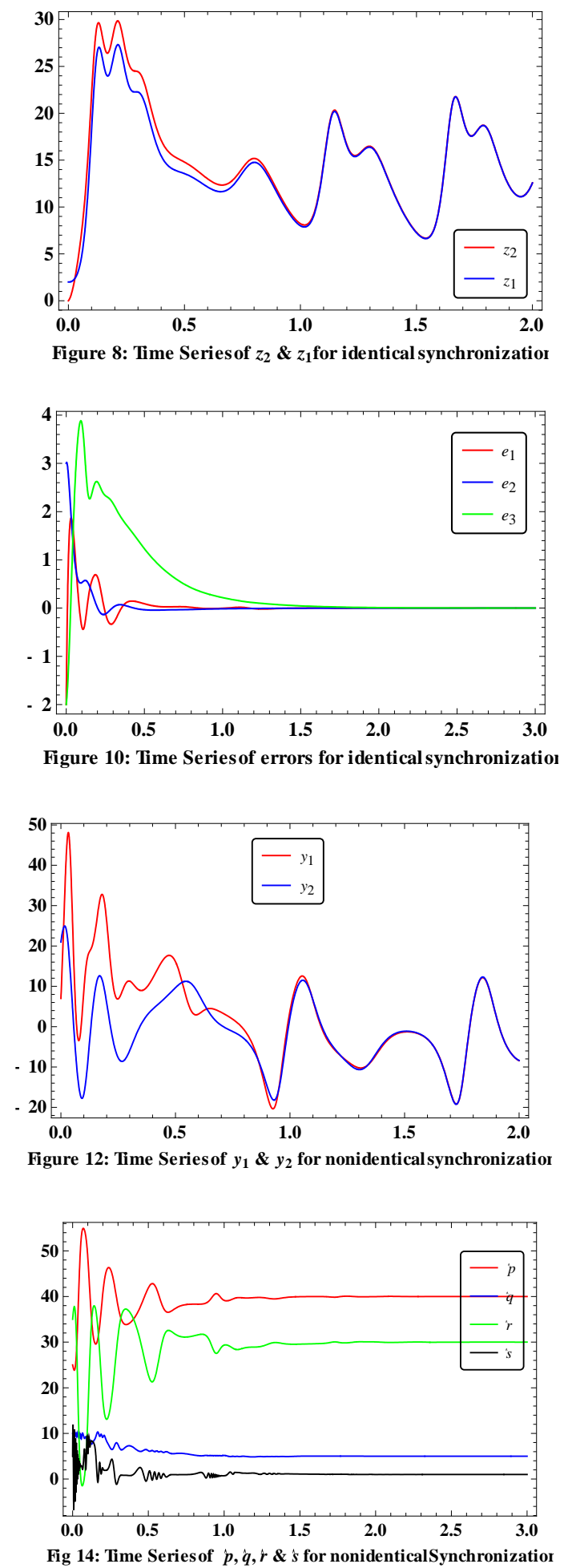

Fig. 15 illustrates the times history of the parameters estimates $p, q, r$, and $s$ and $\alpha, \beta, \gamma$ and $\delta$ respectively of the two nonidentical chaotic systems ([18], [24]) under the update law (36) with initial estimated parameters $\alpha_{1}(0)=5$, $\beta_{1}(0)=10, \gamma_{1}(0)=2$ and $\delta_{1}(0)=1$.

For the two different chaotic systems ([18]) and [24]), that contain parameters mismatches and different structures, the feedback controllers were utilized to synchronize the states of master and slave systems asymptotically when the controls are switched on at $t=0 \mathrm{~s}$. It has been shown that the Liu chaotic system is forced to track the new chaotic system 
and the states of two different chaotic systems show similar behavior, which shows that the error system (Fig. 16) is feedback stabilized and the investigated controllers are more robust to accidental mismatches in the transmitter and receiver. Moreover, Fig. 17 demonstrates derivative of the LEFs of identical chaotic systems [18] and nonidentical chaotic systems ([18] and [24]). The synchronization with negative derivative of the LEFs confirms that the divergence of the flows $(23)$ and $(34)$ are dissipative $(\dot{V}(e)<0)$ that allows large synchronizable interval, which is especially significant for secure communication and engineering applications etc.

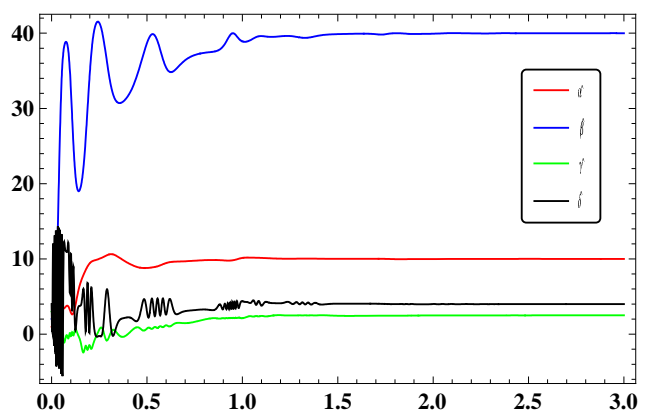

Fig 15: Time Series of $\hat{\imath}, \hat{\beta}, \hat{\jmath} \& \hat{d}$ for nonidenticalSynchronizatiol
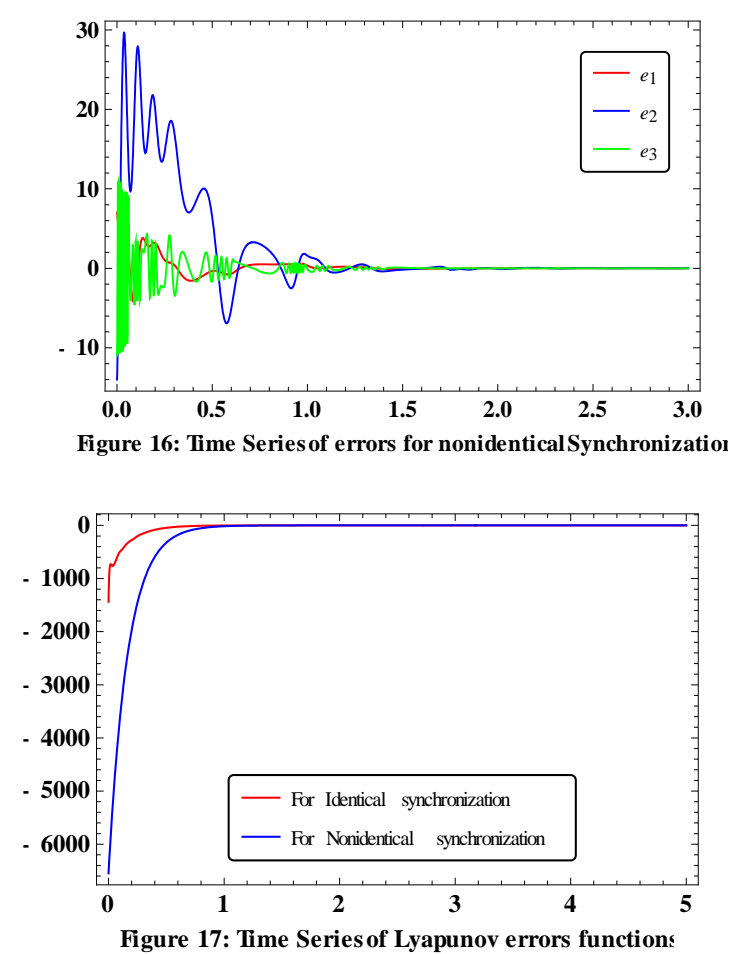

\section{SUMMARY AND CONCLUSION}

In this research, the Adaptive Controllers have been designed to repress the chaos to its equilibrium point at the origin. The synchronization between two identical and two nonidentical 3-D chaotic systems with fully unknown parameters has also been achieved. Based on the Lyapunov Direct Method, the Adaptive control laws for synchronization controllers associated with the Adaptive update laws of system parameters were devised to make the states of two identical and two completely different chaotic systems asymptotically synchronized. The accuracy and efficiency of the proposed controllers have been demonstrated and are validated by the numerical simulations. Furthermore, the synchronization with negative derivative of the PDQLE functions allows substantial synchronizable interval, which is especially important for engineering applications.

One can observe that the convergence of the systems parameters and the synchronization errors are obtained with good accuracy. The chaos control and synchronization of two identical as well as nonidentical chaotic systems presumes potential applications in the field of nonlinear sciences. The obtained results of our current study could to be found in good agreement with experimental observations and can be employed by those researchers involved in the field of secure communication, chaotic modulation and chaotic masking etc.

However, there is always some external disturbances or internal noise exits in real plants that might cause instability and poor performance. Similarly, it is also difficult to determine exactly the values of the system parameters and the controller gain $k$ cannot be properly chosen to guarantee the stability of the controlled system. Hence, future work needs to address these issues to extend the capability of the system and its circuitry design for various applications in different fields such as engineering, hybrid image encryption, secure communications and genetic networks.

\section{ACKNOWLEDGMENT}

The authors are very grateful to the referee(s) for their valuable comments and suggestions which led to an improvement of this research paper.

\section{REFERENCES}

[1] S. Boccaletti, C. Grebogi, Y. C. Lai, H. Mancini, D. Moza, "The control of chaos: theory and applications", Physics reports, vol. 329: pp. 103-197, 2000.

[2] I. Pehlivan, Y. Uyaroglua, "A new chaotic attractor from Genera Lorenz system family and its electronic experimental implementation", Turk Jour. of Elec. Eng. \& Comp. Sci, vol. 18, no. 2, 2010.

[3] E. Ott, C. Grebogi, J. A. Yorke, Controlling Chaos, Phys. Rev. Lett., vol. 64, pp. 1196-1199, 1990.

[4] K. Pyragas, V. Pyragas, I. Z. Kiss, J. L. Hudson, “Adaptive control of unknown unstable steady states of dynamical systems", Phy. Rev. E vol. 70, 2004

[5] L. M. Pecora, T. L. Carroll, "Synchronization in Chaotic Systems", Phys. Rev. Lett., vol 64, no. 8, pp. 821-825, 1990.

[6] A. Saaban, A. Ibrahim, M. Shahzad, I. Ahmad, "Identical Synchronization of a New Chaotic System via Nonlinear Control and Linear Active Control Techniques: A Comparative Analysis", International Journal of Hybrid Information Technology, vol. 7, no. 1, pp. 211-224, 2014.

[7] L. O. Chua, M. Komuro, S. Tanaka, "Simplest chaotic nonautonomous circuit", Phys. Rev. A, vol. 30, pp. 1155-1158, 1984.

[8] I. Ahmad, A. Saaban, A. Ibrahim, M. Shahzad, "Global Chaos Synchronization of Two different Chaotic Systems Using Nonlinear Control", International Journal of Sciences: Basic and Applied Research (IJSBAR), vol. 13, no. 1, pp. 225-238, 2014.

[9] B. Idowu, U. Vincent, A. Njah, "Generalized Adaptive Backstepping Synchronization for Non-identical Parametrically Excited Systems", Nonlinear Analysis: Modelling and Control, vol. 18, no. 1, pp. 112 128,2013

[10] K. S Ojo, A. Njah, S. Ogunjo, "Comparison of backsteeping and modified active control in projective synchronization of chaos in an extended Bonhoffer-van der Pol Oscillator", Pranama Journal of Physics, vol. 80, no. 5, pp 825-835, 2013. 
[11] J. Xing, "Adaptive hybrid function projective synchronization of chaotic systems with fully unknown periodical time-varying parameters", Nonlinear Analysis: Modelling and Control, vol. 18, no. 1, pp 112-128, 2013

[12] S. Vaidyanathen, "Adaptive Control and Synchronization of ShimizuMorioka Chaotic System", International Journal in Foundations of Computer Science \& Technology, vol. 2, no. 4, pp 29-42, 2012.

[13] O. Olusola, "Adaptive Synchronization of identical and Nonidentical Hyperchaotic Systems with unknown Parameters", The African Review of Physics, vol. 7, pp 345-352, 2012.

[14] M. Ali Khan, "Adaptive Synchronization of two coupled NetwonLeipnik System with Uncertain Parameter", Int. Journal of Basic and Applied Sciences, vol. 1., no. 4, pp 439-447, 2012.

[15] S. Vaidyanathen, K. K. Rajagopal, "Global Chaos Synchronization of Hyperchaotic Pang and Hyperchaotic Wang Systems via Adaptive Control", Int. Journal of Soft Computing, vol. 7, no. 1, pp 28-37, 2012.

[16] H. Zhang, X. Ma, B. Xue, "A novel boundedness-based linear and nonlinear approach to control chaos", Chaos, Solitons and Fractals, vol. 22, pp 433-442, 2004

[17] S. Al-Hadhrami, A. Saaban, A. Ibrahim, M. Shazad, I. Ahmad, "Linear Active Control Algorithm to Synchronize a Nonlinear HIV/AIDS Dynamical System", Asian Journal of Applied Sciences and Engineering, vol. 3, no. 2, pp 96-115, 2014.
[18] C. Li, L. Wu, H. Li, Y. Tong, "A novel chaotic system and its topological horseshoe", Nonlinear Analysis, Modelling and Control, vol. 18 , no. 1, pp 66-77, 2013

[19] H. K. Khalil, Non Linear dynamical Systems, Prentice Hall, 3rd Ed, NJ 07458, USA, 2002.

[20] M. Aghababa, H. Aghababa, "Synchronization of nonlinear chaotic electromechanical gyrostat systems with uncertainties", Nonlinear Dyn, vol. 67, pp 2689-2701, 2012.

[21] Z. Xinghua, D. Shougang, "Adaptive Chaos Synchronization for a Type of Non-Smooth-Air-Gap Permanent Magnet Synchronous Motors", Control and Decision Conference, 2008, Ding Shougang, China.

[22] M. Ying-Ying, L. Yun-Gang, "Barbalat's Lemma and its application in analysis of system stability", Journal of Shandong University of Technology, vol. 37, no. 1, pp 51-55, 2007.

[23] L. Nguyen, K. Hong, "Adaptive Synchronization of two coupled chaotic Hindmarsh-Rose Neurons by controlling the membrane potential of a slave neuron", Applied Mathematical Modeling, vol. 37, no. 4, pp 2460-2468, 2013.

[24] T. Zhou, G. Chen, "Classification of Chaos in 3-D Autonomous Quadratic System-1. Basic Framework and Method", International Journal of Bifurcation and Chaos, vol. 16, no. 9, pp 2456-2479, 2006 